\title{
Methotrexate Induced Toxic Epidermal Necrolysis: A Case Report
}

\author{
S Bhuvaneshwari T Shanmugham ${ }^{1}$, C Dinesh M Naidu², Raghuvanshi $\mathbf{V}^{3}$ \\ ${ }^{1}$ Dr. S. Bhuvaneshwari T. Shanmugham, Med. Pharmacology, Assistant Professor, ${ }^{2}$ Dr. C. Dinesh M. Naidu, Professor \\ \& Head, ${ }^{3}$ Dr. Vinay Raghuvanshi, Senior Resident, all authors are affiliated with Department of Pharmacology, \\ Andaman \& Nicobar Islands Institute of Medical Sciences (ANIIMS), Port Blair, India.
}

Address for Correspondence: Dr. S. Bhuvaneshwari T. Shanmugham, Email: nmadhukant7@gmail.com

\begin{abstract}
Methotrexate continues to be one of the most widely used systemic immunosuppressive agents in dermatology. We describe a case of low-dose methotrexate (MTX) toxicity in a patient with chronic plaque psoriasis occurring during long-standing methotrexate therapy. This case report emphasizes the fact that co-medications like NSAIDS may exacerbate MTX toxicity. This patient had a rare methotrexate-induced toxic epidermal necrolysis including cutaneous ulceration. Hence, careful consideration of concomitant medication is required to avoid drug interactions for safe longterm methotrexate treatment.
\end{abstract}

Key words: Methotrexate, Necrolysis, skin, Psoriasis, Toxicity

\section{Introduction}

Toxic epidermal necrolysis (TEN) is a life-threatening disease characterized by extensive destruction of the epidermis. The mortality rate averages $25-30 \%$ due to septicemia and other complications [1]. It is a rare and usually severe adverse reaction to certain drugs. History of medication use exists in over $95 \%$ of patients with TEN. The drugs most implicated in TEN are antibiotics such as sulfonamides, nonsteroidal anti-inflammatory drugs (NSAIDS), allopurinol, antimetabolites (methotrexate), antiretroviral drugs, and anticonvulsants such as phenobarbitone, phenytoin, carbamazepine, and valproic acid [2]. There are reports of MTX induced TEN due to high dose of MTX but TEN like presentation with low dose of MTX has rarely been reported and that too in a case of psoriasis [1].

Methotrexate continues to be one of the most widely used systemic immunosuppressive agents in dermatology [3]. Here, we report a case where a patient presented with TEN, even on low dose MTX therapy.

\section{Case Report}

A 49 years old female patient, resident of Port Blair, Andaman and Nicobar Islands presented to the female ward with complaints of ulcer on dorsum of left foot, blisters, erythematous eruptions all over body and fever since 1 week. Patient is a known case of chronic plaque psoriasis and is on methotrexate $15 \mathrm{mg} /$ week since 2 years. She gives history of taking analgesic ibuprofen for body ache. The skin lesions developed approximately after 24 hours of taking NSAID ibuprofen. Patient also developed erythematous painful swelling on face, upper limbs and lower limbs with subsequent bullous lesions on those sites. Oral mucosa showed the presence of blisters. (Fig.1, Fig.2, Fig.3). A positive Nikolsky's sign (denudation of the skin with gentle tangential pressure) was also present. Diagnosis of toxic epidermal necrolysis was made based on the clinical examination. Symptoms and signs resolved within 2 weeks following withdrawal of methotrexate, supportive treatment and appropriate skin care. Routine blood parameters were within normal limits except for uncontrolled diabetes. Accordingly patient was started with insulin preparations. Rest of the systemic examination was unremarkable. Patient was treated successfully with conservative measures and discharged after 2 weeks and advised for regular follow-up.

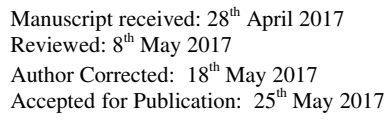




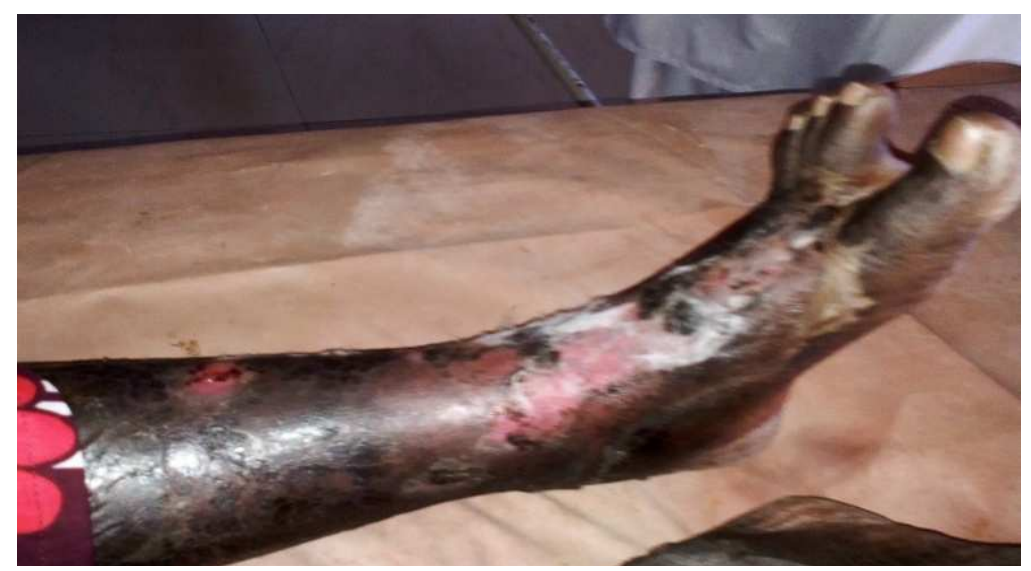

Fig-1: Cutaneous ulceration involving lower limbs

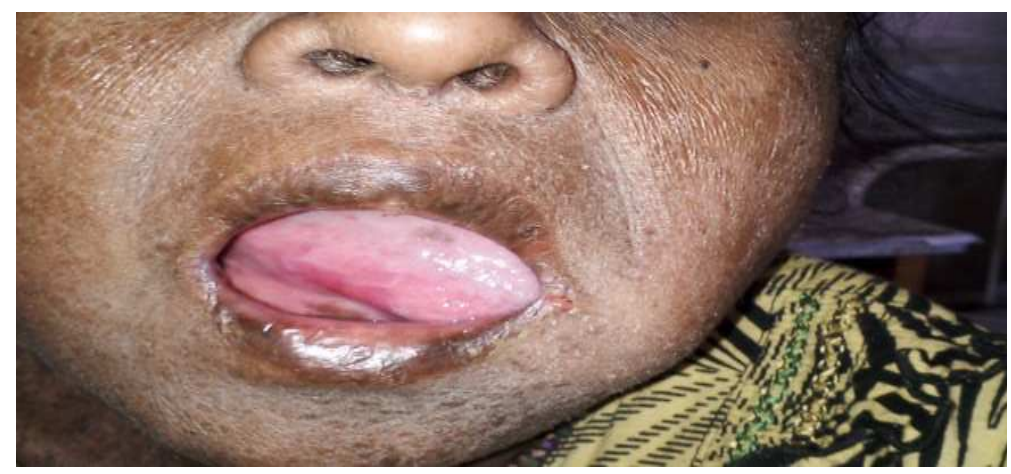

Fig.-2: Swelling of face with erythematous areas \& oral erosions

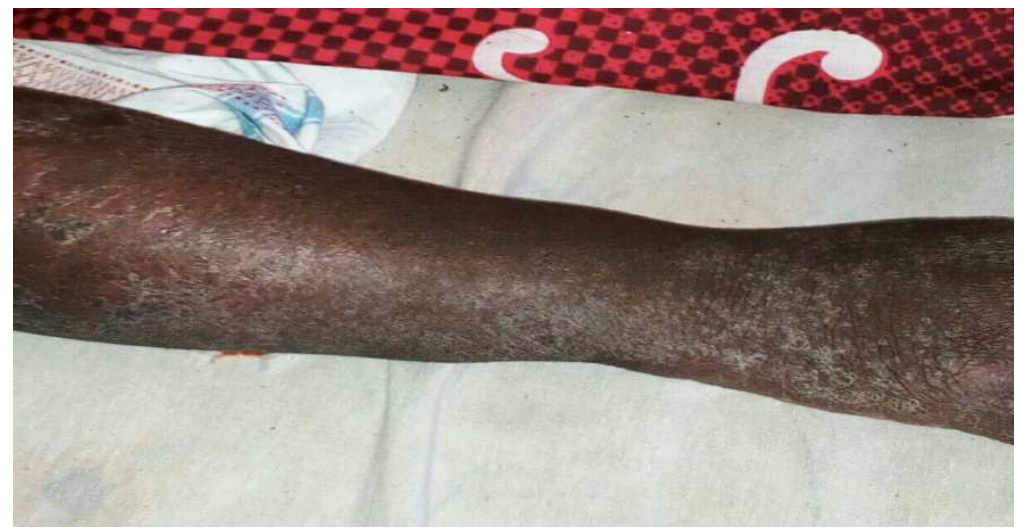

Fig.-3: Skin lesions involving upper limb

\section{Discussion}

Several drugs may influence methotrexate metabolism and/or potentiate methotrexate-induced toxicity, including some nonsteroidal anti-inflammatory drugs. Lawrence and Dahl (1984) described seven patients who developed skin ulceration on psoriatic plaques and pre-existing dermatitis after a low dose of MTX. Four of these patients received long-term MTX therapy and ulceration occurred after increases in MTX dosage or after taking nonsteroidal anti inflammatory drugs
(NSAIDs). All of these patients were receiving NSAIDs when skin ulceration occurred, and the ulceration of five patients healed after reducing the MTX dosage [4]. Similarly this case on low dose methotrexate also presented with skin ulceration and lesions after taking NSAID. The most likely etiology for TEN in this case was thus methotrexate. Patients and physicians should be aware of possible drug interactions and regularly review additional medication use. 


\section{Conclusion}

This rare case has been presented to make us aware about this occasional life-threatening adverse effect of MTX which might be due to drug interaction.

Hence, careful consideration of concomitant medications is required to avoid drug interactions for safe long-term methotrexate treatment [5].

Declaration of patient consent- The authors certify that they have obtained all appropriate patient consent forms. In the form the patient has given her consent for her images and other clinical information to be reported in the journal. The patient understands that her names and initials will not be published and due efforts will be made to conceal identity.

\section{Funding: Nil, Conflict of interest: None Permission of IRB: Yes}

\section{References}

1. Sancheti K, Podder I, Gharami RC. Toxic epidermal necrolysis like reaction due to low-dose methotrexate in a case of cutaneous lupus erythematosus: A rare occurrence. Indian J Dermatol 2016; 61(1): 102-4.
Available from: http://www.e-ijd.org/text.asp 2016/61 /1/ 102/17405

2. Tiwari P, Panik R, Bhattacharya A, Ahirwar D, Chandy A. Toxic epidermal necrolysis: an update. Asian Pac J Trop Dis 2013; 3(2): 85-92.doi:10.1016 /S2222-1808 (13)60051-1

3. Weidmann A, Foulkes AC, Kirkham N, Reynolds NJ. Methotrexate toxicity during treatment of chronic plaque psoriasis: a case report and review of the literature. Dermatology and therapy 2014;4(2):145-156. doi:10.1007/s13555-014-0056-z.

4. Tazi I, Madani A, Zafad S, Harif M, Quessar A, Benchekroun S. Methotrexate-induced toxic epidermal necrolysis: A case report. Int J Med Med Sci. 2009; 1(4): 99-101. Available online at http: //www. academicjournals.org/IJMMS

5. Naldi L, Griffiths CEM. Traditional therapies in the management of moderate to severe chronic plaque psoriasis: An assessment of the benefits and risks. Br J Dermatol. 2005;152(4):597-615. doi: 10.1111/j.13652133.2005.06563.x.

\section{How to cite this article?}

S Bhuvaneshwari T Shanmugham, C Dinesh M Naidu, Raghuvanshi V. Methotrexate Induced Toxic Epidermal Necrolysis: A Case Report. Int J Med Res Rev 2017;5(05):492-494. doi:10.17511/ijmrr. 2017.i05.09. 